Natural Hazards and Earth System Sciences (2001) 1: 127-136

(C) European Geophysical Society 2001

Natural Hazards and Earth System Sciences

\title{
Study of electromagnetic emissions associated with seismic activity in Kamchatka region
}

\author{
V. Gladychev ${ }^{1}$, L. Baransky ${ }^{1}$, A. Schekotov ${ }^{1}$, E. Fedorov ${ }^{1}$, O. Pokhotelov ${ }^{1}$, S. Andreevsky $^{1}$, A. Rozhnoi ${ }^{1}$, \\ Y. Khabazin ${ }^{1}$, G. Belyaev ${ }^{1}$, A. Gorbatikov ${ }^{1}$, E. Gordeev ${ }^{2}$, V. Chebrov ${ }^{2}$, V. Sinitsin ${ }^{2}$, A. Lutikov ${ }^{3}$, S. Yunga ${ }^{3}$,

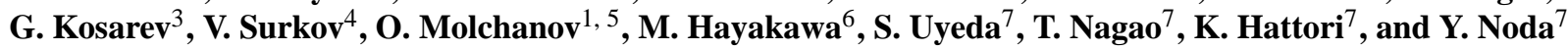 \\ ${ }^{1}$ Institute of Physics of the Earth Russian Academy of Sciences, 10, Bolshaya Gruzinskaya, 123995 Moscow D-242, Russia \\ ${ }^{2}$ Institute of Geophysical Survey, Russian Academy of Sciences, Far-East Branch, Petropavlovsk-Kamchatski, Kamchatka, \\ Russia \\ ${ }^{3}$ Institute of Geophysical Survey, Russian Academy of Sciences 10, Bolshaya Gruzinskaya, 123995 Moscow d-242, Russia \\ ${ }^{4}$ Moscow State Engineering Physics Institute, 31, Kashirskoye road, 115409 Moscow, Russia \\ ${ }^{5}$ Earth Observation Research Center, NASDA, Roppongi 1-9-9, Minato-ku, Tokyo 106, Japan \\ ${ }^{6}$ Department of Electronics Engineering, The University of Electro-Communication, 1-5-1, Chofugaoka, Chofu-shi, Tokyo \\ 182, Japan \\ ${ }^{7}$ RIKEN International Frontier Research Group on Earthquakes at Earthquake Prediction Research Center, Tokai University, \\ 3-20-1, Orido, Shimizu,424-8610, Japan
}

Received: 5 June 2001 - Revised: 5 October 2001 - Accepted: 8 January 2001

\begin{abstract}
A review of data processing of electromagnetic emission observation collected at the Complex Geophysical Observatory Karimshino (Kamchatka peninsula) during the first 5 months (July-November, 2000) of its operation is given. The main goal of this study addresses the detection of the phenomena associated with Kamchatka seismic activity. The following observations have been conducted at CGO: variations of ULF/ELF magnetic field, geoelectric potentials (telluric currents), and VLF signals from navigation radio transmitters. The methods of data processing of these observations are discussed. The examples of the first experimental results are presented.
\end{abstract}

\section{Introduction}

There was a great deal of research during the last few years devoted to the analysis of electromagnetic and acoustic emissions associated with the earthquake (hereafter EQ) preparation. It is worth mentioning that in the past the data processing, relevant to this problem, was limited as a rule to the analyses of separate geophysical fields. No complex analysis involving the cross-correlation study has been used. Moreover, the manmade background provided a substantial screening of the effects due to the earthquake preparation processes. Thus, in order to obtain reliable results, it is necessary to perform the long-term observations of a large number of various

Correspondence to: V. Gladychev (glad@uipe-ras.scgis.ru) geophysical parameters in a region with enhanced seismic activity and low industrial noise. In that respect Kamchatka peninsula is an ideal place for the installation of the Complex Geophysical Observatory (hereafter CGO), which yields these requirements.

The observatory has been established by Russian and Japanese scientists during a scientific campaign in 1999 in addition to an existing network of seismic and geophysical stations. It is located in Karimshino $\left(52.94^{\circ} \mathrm{N}, 158.25^{\circ} \mathrm{E}\right.$, $L=2.1)$ at a distance of about $50 \mathrm{~km}$ from PetropavlovskKamchatsky. No pronounced sources of industrial noise are found close to this particular region. The main purpose of our study is to provide analysis of the correlation of seismic activity with electromagnetic phenomena (e.g. the change in the ULF/ELF magnetic field, telluric currents, VLF signals from navigation radio transmitters) and acoustic emission. The measuring devices and digital registration systems placed at this observatory, as well as the seismic activity of the Kamchatka region, are described by Uyeda et al. (2001).

\section{Seismological data}

Simultaneous observation of seismic and electromagnetic phenomena carried out at the same place gives us a unique possibility to find a correlation between the temporal variations of local seismicity and the change in the Earth's magnetic field. Regarding the case study or analysis of the individual precursory event, it is important to understand how to 
(a)

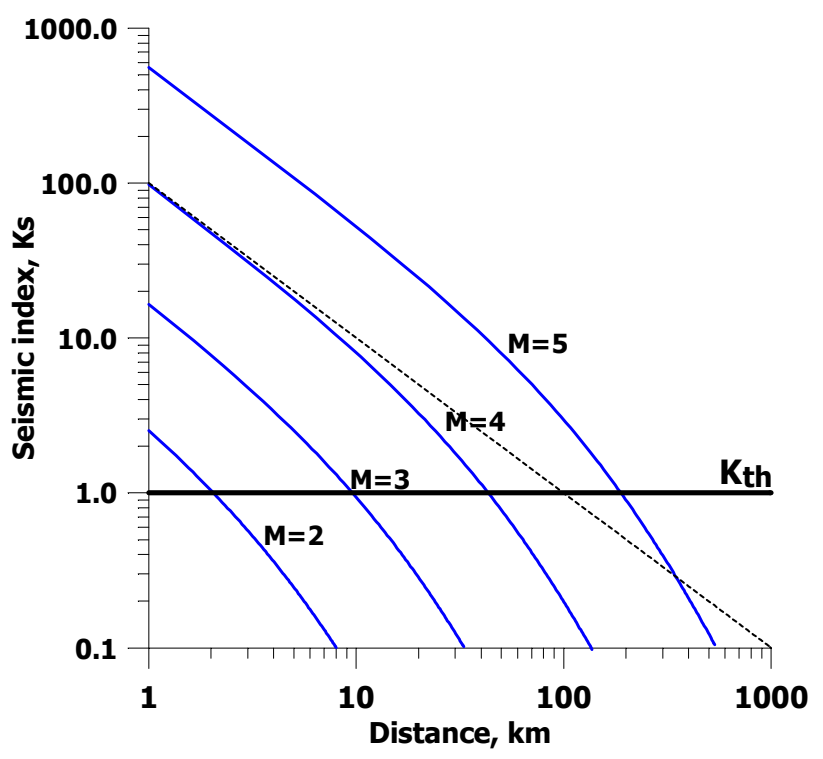

(b)

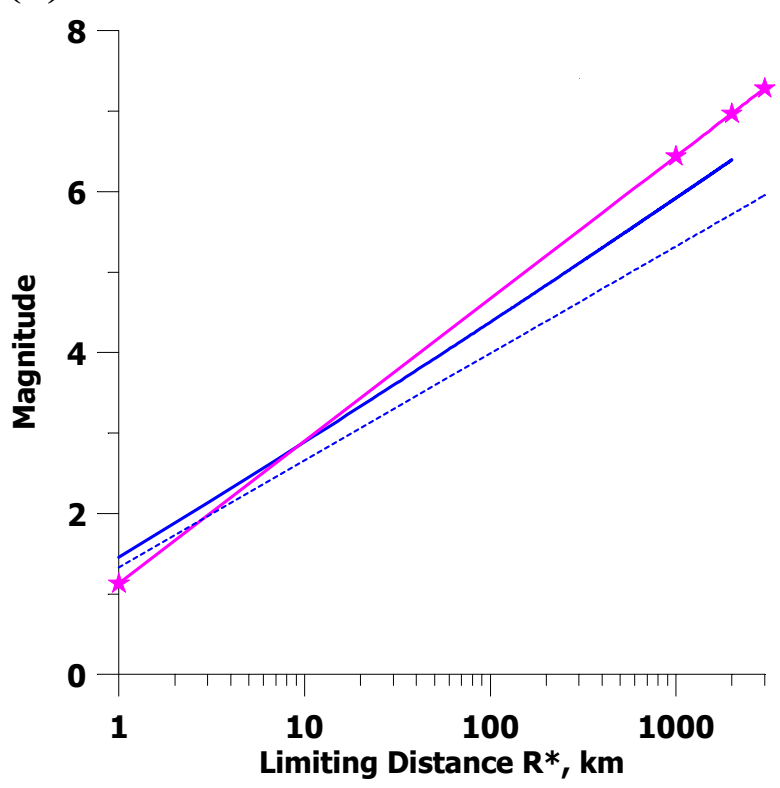

Fig. 1. (a) $K s(R)$ dependence. Dashed line is a simplified dependence for $M s=4$ when an attenuation $(F a=1)$ is neglected. (b) Magnitude-maximal distance relation $M s\left(R^{*}\right)$ from $A E$ observations (Eq. 1), line with stars; and for our relationship (Eq. 3), solid line. Dashed line is for the simplified dependence.

estimate the intensity of the seismic shock for precursor production. To estimate the effectiveness of the seismic shock for electromagnetic precursor production, the special $K s$ index was introduced. The data of the Kamchatka regional network of seismic stations were used for the estimation of the $K s$-index.

Recently, Gorbatikov et al. (2000) has analyzed the acoustic emission precursors at Matsushiro station. These authors have assumed that seismic response is proportional to $\frac{\sqrt{E s}}{R^{\alpha}}$, where $E s$ is the energy of the seismic shock $\left(E s \sim 10^{3 / 2 M s}\right.$ ), $R$ is the distance between EQ hipocenter and observational point, and coefficient $\alpha \geq 1$ describes the mutual influence of the seismic energy divergence and the attenuation. As a result, they have presented the following limiting condition:

$M s=1.33 \alpha \log R^{*}+B$

where $R^{*}$ is limiting distance for EQ with magnitude $M s$. The values for $\alpha=1.33$ and $B=1.13$ have been estimated directly from the observational data.

Here, the same approach has been used assuming that "seismic index" $K s$ is proportional to $\sqrt{P s}$, where $P s$ stands for the seismic energy flux. Then, we have made use of the results provided by Molchanov et al. (2001) on the presentation of the seismic attenuation function $F a(R / R a)$, where $R a$ is the attenuation distance (see Fig. 2). We have assumed the following relationship to be valid:

$$
\begin{gathered}
K s=K \operatorname{th} 10^{0.75 M s} /\left(10 R^{*}\right) F a, \\
F a=\left(1+R^{*} 10^{-M s / 2}\right)^{-2.33}
\end{gathered}
$$

and assumed that the threshold level is $K t h=1$, i.e. $\max$ imal distance for seismic shock with $M s=4$, is about $100 \mathrm{~km}$. Dependence of $K s(R)$ for the different $M s$ values is shown in Fig. 1a. As before, it is easy to find the relationship for the limiting distance:

$M s=1.33 \log \left(10 R^{*} / F a\left(R^{*} / R a\right)\right)$.

It is shown in Fig. 1b, along with other previous relationships. General similarity of these curves leads to a conclusion that our choice of $K t h=1$ criterion is rather justified. It means that every seismic event with $K s>1$ can be considered as a seismic precursor.

Figure 2 shows some characteristics (including $K s$-index) of seismic activity in the Kamchatka region for EQ's with $M s \geq 3$ within the time domain 24 June $2000-27$ August 2000 .

\section{ULF/ELF magnetic field variations}

During the last 10 years, the experimental evidence of the existence of earthquake precursors in the ULF/ELF magnetic field has been reported, e.g. EQs in Loma Prieta, California (Bernardi et al., 1991), Guam Island (Hayakawa et al., 1996a), Spitak and Racha in Caucasus (Kopytenko et al., 1993). Several EQs in Japan were preceded by the anomalous ULF magnetic signals. All of these EQs had a magnitude $M \geq 6.1$ and the maximum amplitude of the ULF precursor signal varied drastically depending on the distance $R$ of the observational site from the EQ epicenter: $6 \mathrm{nT}$ for Loma Prieta $(R=7 \mathrm{~km})$ and $0.1-0.2 \mathrm{nT}$ for Spitak $(R=128 \mathrm{~km})$. No precursors have been detected prior to the EQs with $R \geq 130 \mathrm{~km}$ and $M \geq 6$. Observations of ULF signals preceding the Spitak, Guam and Japanese Eqs have 


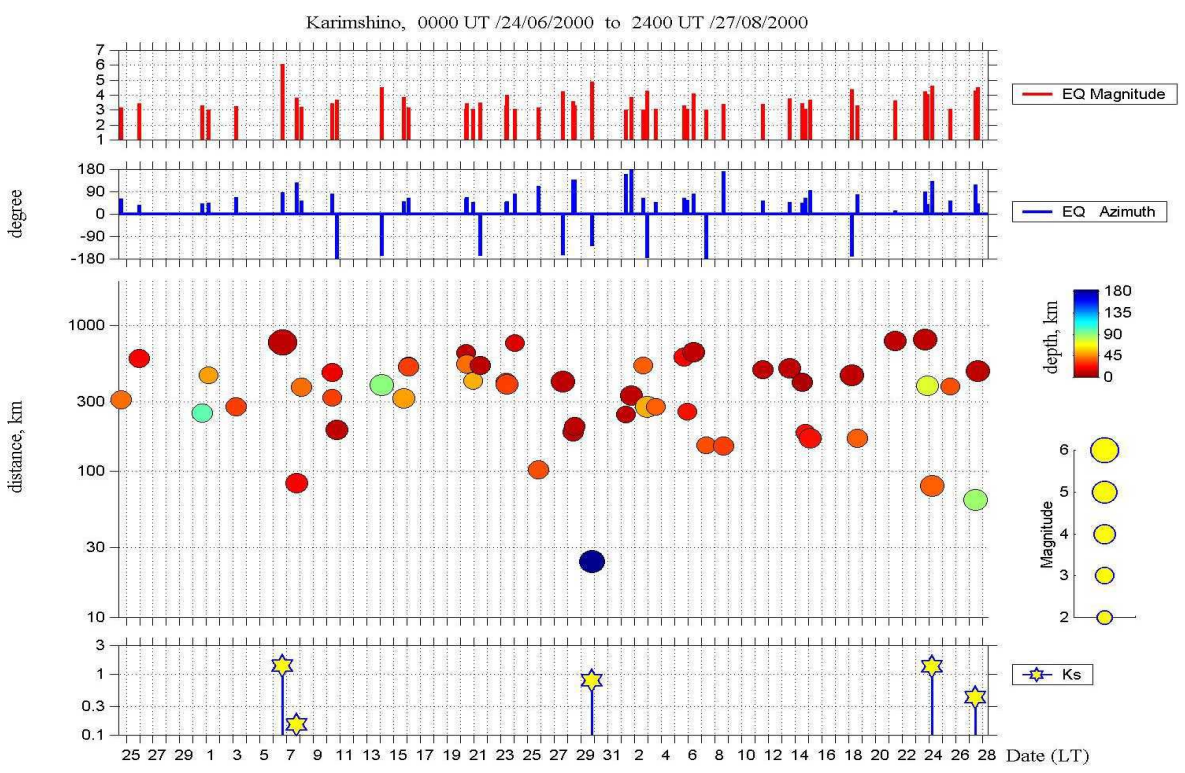

Fig. 2. Seismic activity in Kamchatka region for EQ with $M s \geq 3$ in time interval 24 June 2000 - 27 August 2000. From top to bottom: EQ magnitude $M$; azimuth of direction toward EQ epicenter from CGO counted out from the north direction clockwise (+) or counterclockwise $(-)$; distance $D$ between EQ epicenter and Karimshino (depth of EQ hypocenter and EQ magnitude are indicated to the right from the panel); value of $K s$.
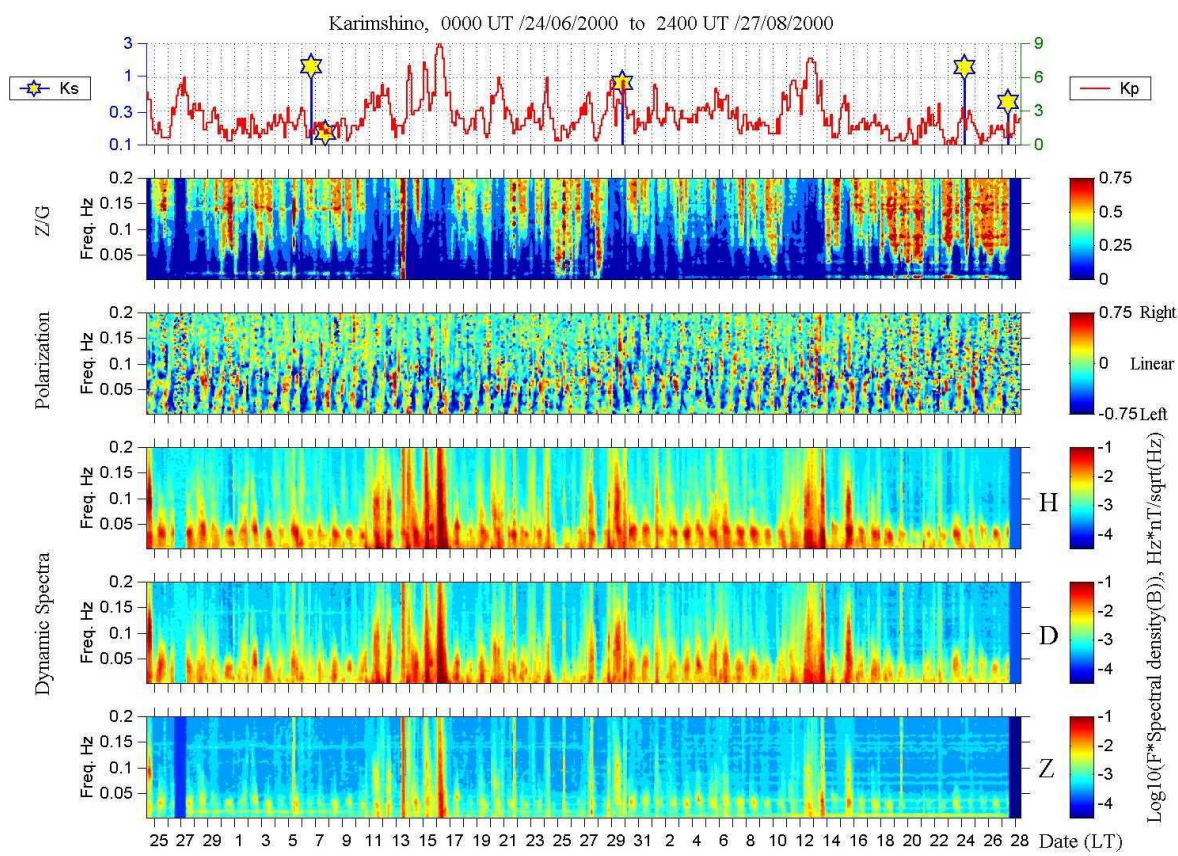

Fig. 3. Dynamic spectra for time interval 24 June $2000-27$ August 2000 in frequency band $0.0025-0.2 \mathrm{~Hz}$. Panels from top to bottom display the following parameters: $K p$, and $K s$, dynamic spectra of $Z / G$ ratio, of ellipticity in horizontal plane and of $H, D$ and $Z$ components of the magnetic field.

displayed anomalous values of vertical to horizontal component ratios $Z / H, Z / D$ or $Z /\left(\sqrt{H^{2}+D^{2}}\right)=Z / G$. One may assume that this feature is typical for magnetic pulsations of lithosphere origin and can be used as a distinctive characteristic of seismogenic signals. At present, very little information is available on precursor signal in the ELF fre- quency range (e.g. Dea et al., 1993).

Considering the peculiarities of the Kamchatka seismic activity (Uyeda et al., 2001) and the experimental data related to ULF/ELF precursors, a basic and rather difficult problem of the search for EQ precursor is their distinction from the natural background. The favourable factor for detection of 


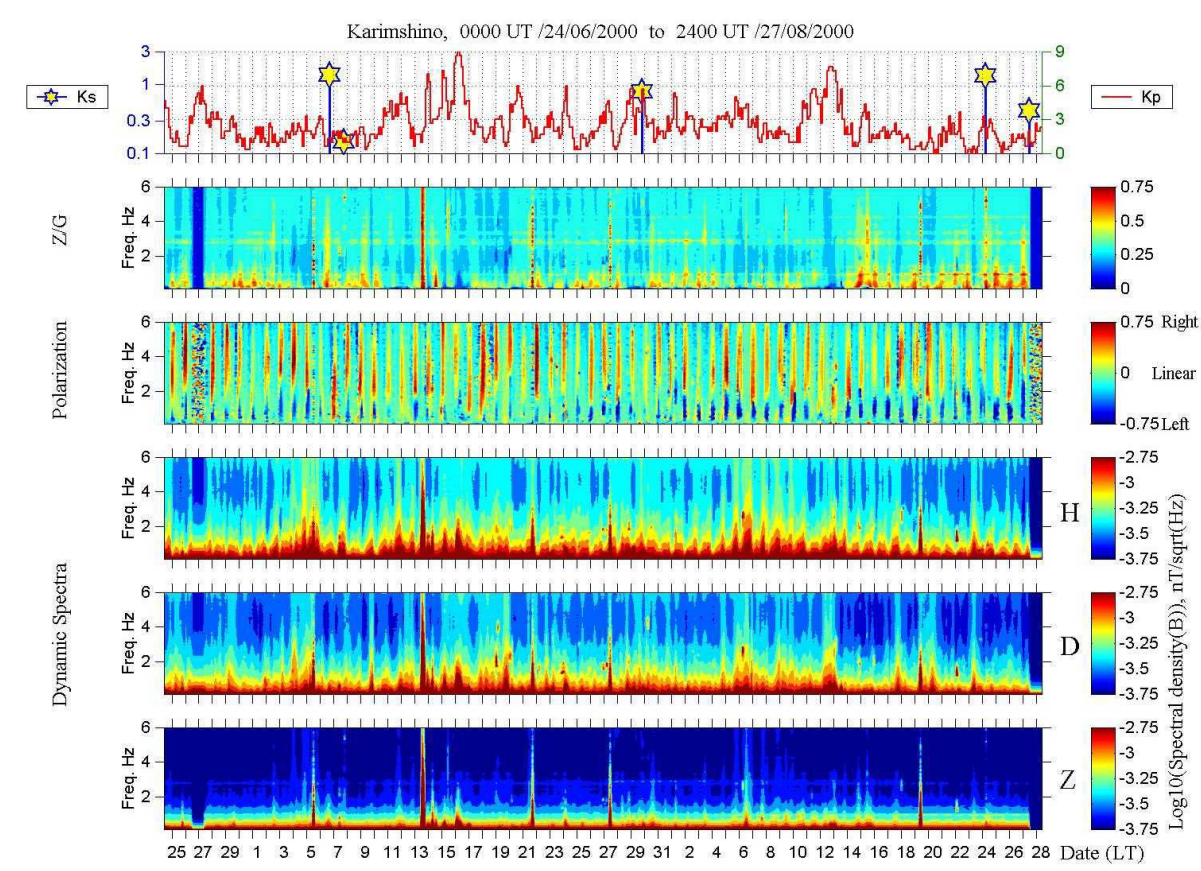

Fig. 4. The same as in Fig. 3 but for frequency band $0.1-6 \mathrm{~Hz}$.

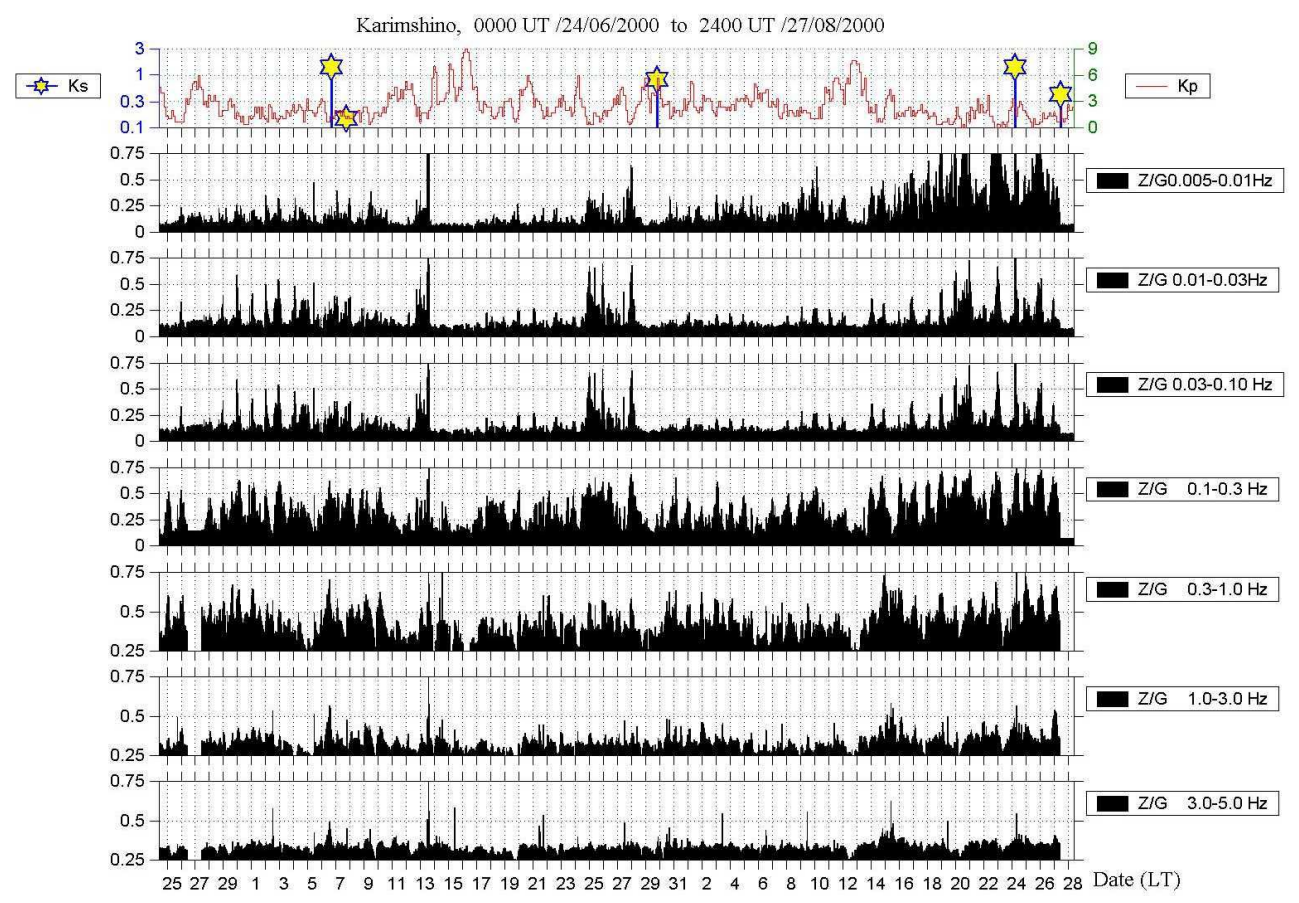

Fig. 5. The behaviour of magnetic field components ratio $Z / G$ in different frequency bands $(0.005-5.0 \mathrm{~Hz})$ for the time interval $24 \mathrm{June}$ 2000 - 27 August 2000.

the EQ precursor is related to the presence of very weak industrial noise.

Taking this into account, one may propose the possible methods of EQ precursor detection.

1. It is useful to take an advantage of high homogeneity of the ULF/ELF background field (its attenuation
$A \leq 3-4 \mathrm{db} / 1000 \mathrm{~km})$ in comparison with the field of EQ precursor $(A \geq 30 \mathrm{db} / 100 \mathrm{~km})$. Taking this fact into account one can compare the magnetometer data of Karimshino and neighbouring stations located along the same geomagnetic meridian $210^{\circ}$ (e.g. Magadan or conjugate station Canberra). This comparison facilitates 


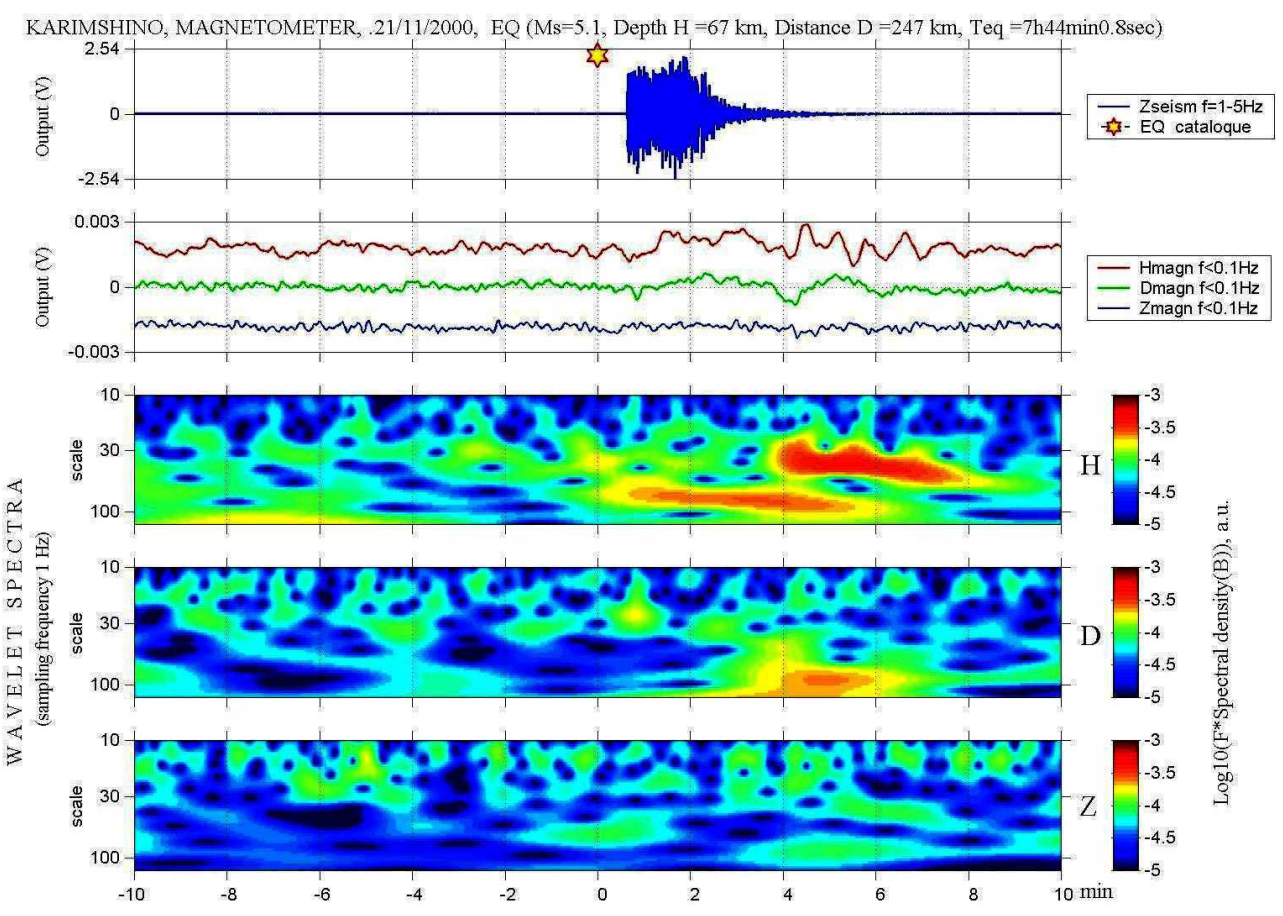

Fig. 6. EQ and Wavelet analyses of related magnetic field perturbations. From top to bottom: - Seismometer signal; - $H, D, Z$ magnetic component response to the seismic waves; Wavelet spectra of the same components, which are calculated with programs of Wave Lab. 802. In this figure time is counted in min from the EQ moment, which is marked by the star in the upper panel. The frequency of the spectral components is roughly in proportion to inverted scale $(100 \approx 0.01 \mathrm{~Hz})$.

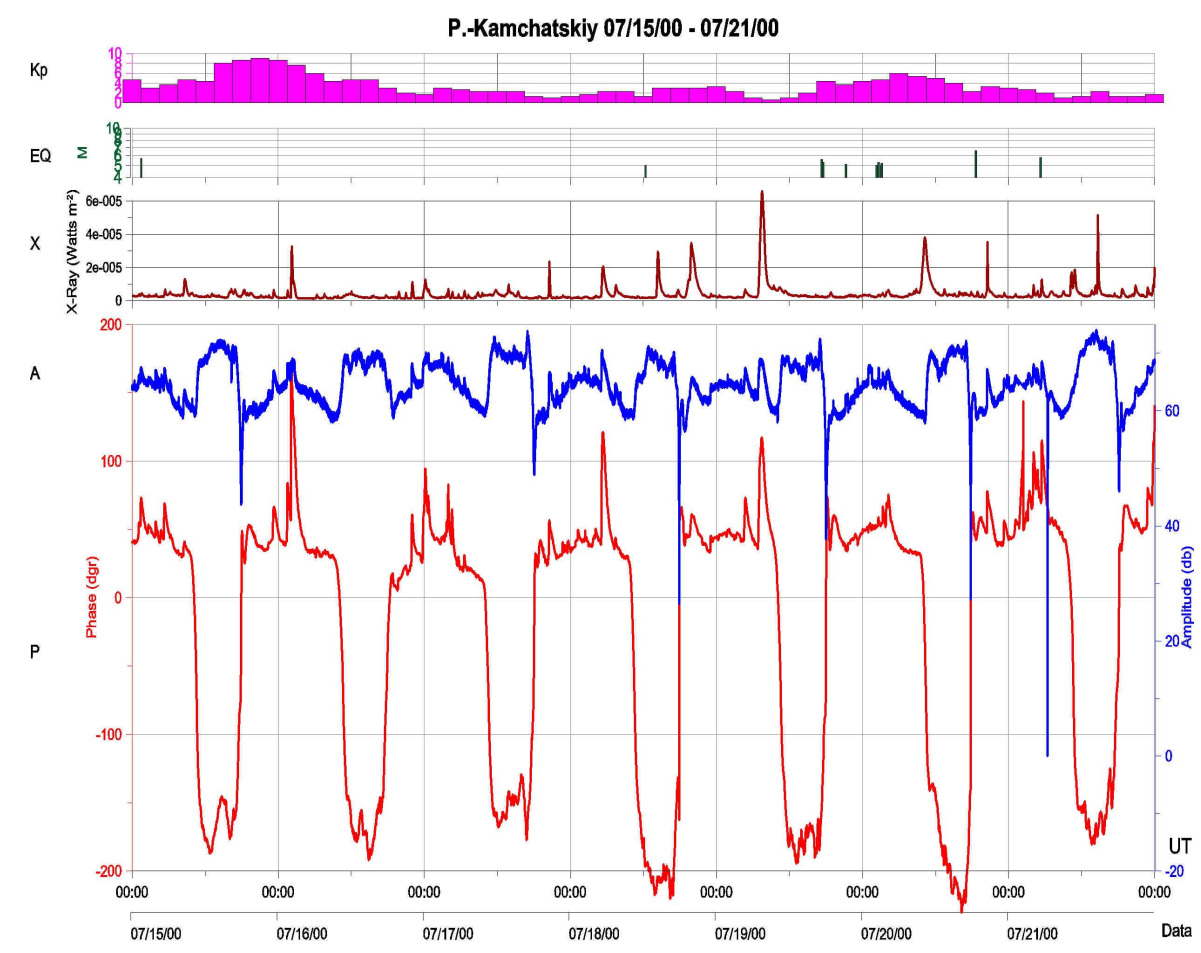

Fig. 7. From top to bottom: (1) planetary index of magnetic field disturbance $K p$, (2) histogram of EQ in a given area stretched along wave path Japan-Kamchatka and has the width of two Frenel's zones, (3) the X-ray flashes (data from GEOS-10 satellite) (4), (5) the records of $A$ and $P$ of VLF signal (the last one after restoration of $P$ continuity) for wave path Japan-Kamchatka with distinct diurnal variation of these values. One can see that the X-ray bursts are at almost the same time as impulses of $A$ and $P$ signals. 
the detection of anomalous signals, which is abnormal in the Karimshino background field and therefore can be considered as a probable EQ precursor. It is more effective if the distance between magnetometers is about tens of $\mathrm{km}$ (the gradient method).

2. Geomagnetic pulsations show rather pronounced diurnal periodicity that can be revealed in the fairly definite local time intervals of occurrence for each type of pulsation within the characteristics of this type of frequency band (Pudovkin et al., 1976). Such a property of geomagnetic pulsations assumes the existence of frequency bands and intervals of local time (frequency-time "windows"), where the permanent background of magnetosphere origin has a relatively stable low level. Therefore, the search for EQ precursors in such "windows" is rather promising. Calculation of averaged diurnal dynamic spectra of pulsations can serve as an efficient guide for the detection of these "windows".

3. According to Hayakawa et al. (1996), an effective method of EQ precursor detection can be the monitoring of polarization characteristics of ULF emissions, particularly such as the ratios $Z / H, Z / D$ or $Z / G$.

4. For the ELF band, the promising method for the search for an EQ precursor can be the monitoring of intensities in frequency gaps between different Schumann resonances. More practical is the monitoring of the ratio of intensities of Schumann resonances.

The last 3 methods are realized first of all by calculations of dynamic spectra for:

- Intensities of $H, D, Z$ components,

- The $Z / G$ ratio,

- Two polarization parameters: (a) the ratio of minor to major axis of polarization ellipse in horizontal plane (ellipticity), which varies from -1 (left-hand circular polarization) through 0 (linear polarization) to +1 (righthand circular polarization) and (b) the angle of major axis of polarization ellipse in horizontal plane from northward direction, which varies from $-90^{\circ}$ (East) to $+90^{\circ}$ (West).

The latter characteristics can also be calculated in the vertical plane. Taking into account the broad frequency range, all of these dynamic spectra are calculated separately for three frequency bands: $0.0025-0.2 \mathrm{~Hz}, 0.1-6 \mathrm{~Hz}, 6-24 \mathrm{~Hz}$ and are shown as color plots (for different levels of parameters under study) with frequency-local time coordinate axes. The averaging was performed over three-hour intervals for all spectral values. Such pictures can cover one day, week or the entire month, according to our selection.

Dynamic wave spectra for the time interval 24 June 2000 - 26 August 2000 and frequency bands $0.0025-0.2 \mathrm{~Hz}$ and $0.1-6 \mathrm{~Hz}$ are shown in Fig. 3 and Fig. 4, respectively. They can serve as an example of our first study of general regularities of the field and detection of "windows". It is necessary to note that in Fig. 3 for $H, D$ and $Z$ components, the values $\log _{10}(F \times$ Spectral density $(B), \mathrm{Hz} \times \mathrm{nT} / \mathrm{sqrt}(\mathrm{Hz}))$ are calculated, whereas in Fig. 4, for the same components, the values $\log _{10}$ (Spectral density $(B), \mathrm{nT} / \mathrm{sqrt}(\mathrm{Hz})$ ) are calculated.

Using the data similar to those shown in Figs. 3 and 4, one may arrive at the following conclusions:

1. The approximate inverse proportion of the $Z / G$ ratio to $K p$-index is well observed for frequencies up to $\sim 0.3-$ $1 \mathrm{~Hz}$.

2. The diurnal variation of ellipticity can be noticed with a left-hand rotation of the total horizontal vector at the forenoon hours and right-hand rotation in the afternoon. This diurnal variation is the most distinct for frequencies $\sim 0.003-0.1 \mathrm{~Hz}$, i.e. for Pc5 - Pc3 pulsations. These pulsations show rather regular behaviour (Samson et al., 1971; Ansari and Fraser, 1986). Careful investigation all these variations is assumed in order to exclude them and obtain a more clear background in order to distinguish anomalous behaviour of ellipticity.

3. A diurnal variation of intensity is observed for all ULF emissions up to $\sim 0.2-1 \mathrm{~Hz}$ with nighttime minimum and daytime maximum. The intensity of emissions also increases with the growth of the $K p$-index. The latter dependence is more pronounced than the diurnal. But at frequencies higher than $\sim 0.2 \mathrm{~Hz}$, the dependence of intensity on $K p$-index vanishes.

4. For $K p \leq 2$, the frequency "window" occupies the frequency band $0.02-6 \mathrm{~Hz}$ and the interval of local time around 24:00 LT. During daytime, the frequency range of the "window" is narrower and occupies the 0.05$6 \mathrm{~Hz}$ frequency band. This "window" must be supplemented by the frequencies, which are disposed between frequencies of Schumann resonances $(\sim 9-13 \mathrm{~Hz}, 16-$ $19 \mathrm{~Hz}$ etc.). For $K p>2$, the lower frequency boundary of the "window" increases up to $\sim 0.1-0.2 \mathrm{~Hz}$ and becomes independent on local time.

The EQ precursor search in the frequency band $0.01 \pm 0.003 \mathrm{~Hz}$, where anomalous behaviour of $Z / G$ has been observed recently by Uyeda et al. (2000) a few weeks before an EQ, can also be considered as promising.

For more detailed data analysis, it is necessary to measure all the enumerated above parameters (intensity of $H, D, Z$ components, $Z / G$ etc.) in rather narrow frequency bands, which involves the detected frequency "windows".

However, at the first steps of investigation, the whole frequency range under study was analyzed in rather broad frequency bands with $\Delta f / f \approx 1$ (Fig. 5) (the effectiveness of EQ precursor searching using this method was demonstrated by Bernardi et al., 1991). Figure 5 shows the variation of the $Z / G$ ratio in different frequency bands $(0.005-5.0 \mathrm{~Hz})$ for the same time interval 24 June $2000-27$ August 2000. A roughly inverse relation of the $Z / G$ value to the $K p$-index 


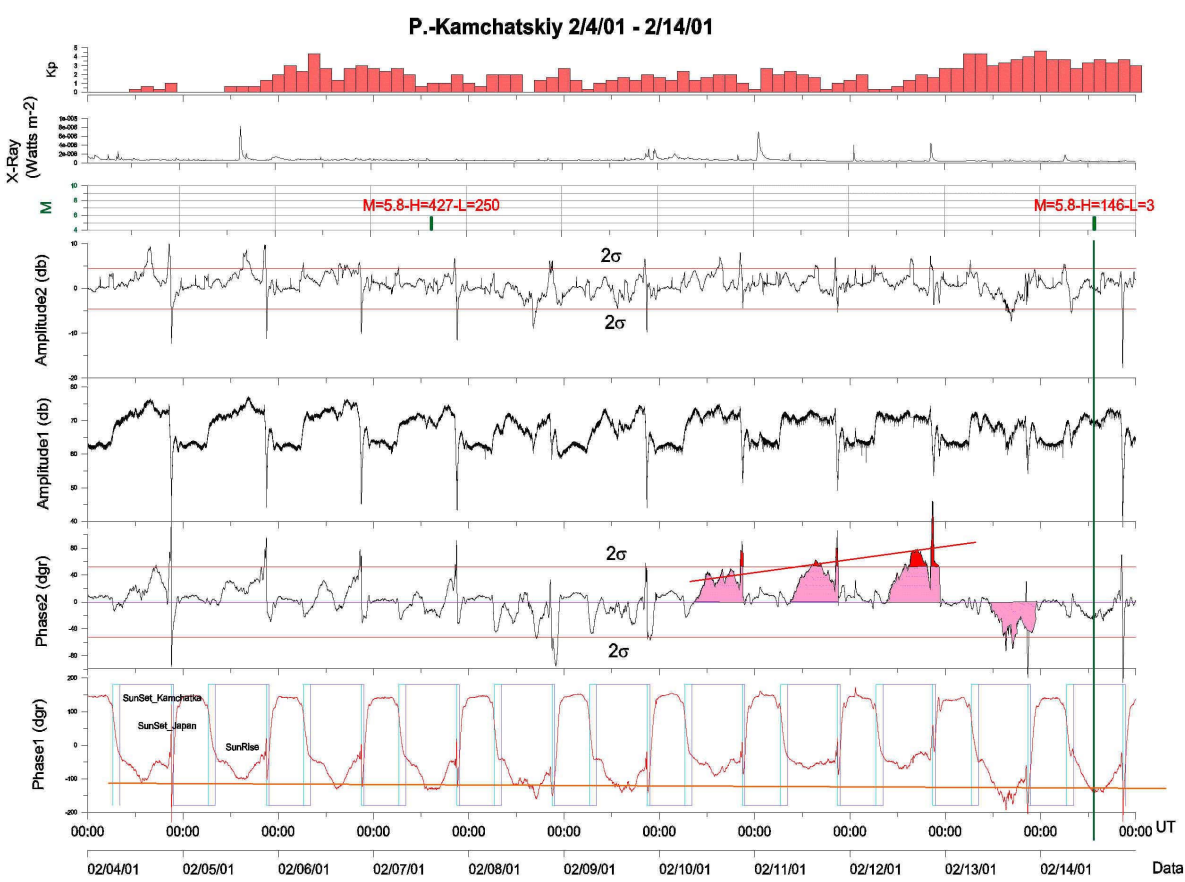

Fig. 8. From top to bottom: (1) planetary $K p$-index, (2) X-ray flashes, (3) histogram of EQ in the area, which is stretched along wave path Japan-Kamchatka (width of two Frenel's zones), (4), (5) the records of $A$ for the same wave path received after all steps of data processing (Amplitude 2) and before the subtraction of its averaged diurnal variation (Amplitude 1), (6), (7) the records of $P$ after all steps of data processing (Phase 2) and before subtraction of its averaged diurnal variation (Phase 1). Vertical dotted lines in Phase 1 mark Terminators.

can be noticed for frequencies up to $\sim 0.3-1 \mathrm{~Hz}$. (similar to that shown in Figs. 3 and 4).

Finally, we make an attempt to estimate the possibility of a supplementary method of signal processing for the signal detection associated with EQ (Fig. 6).

One can see the appearance of the $H$ component emission in the frequency band $0.01-0.03 \mathrm{~Hz}$ at the moment of EQ. The emission is lasting some time after the seismic signal has vanished.

\section{Observations of VLF signals from radio transmitters}

The purpose of observations is to investigate the influence of seismic activity along the paths of signal propagation on the measured values of the amplitude and phase of the VLF signals. In the paper by Uyeda et al. (2001) the map of radio transmitters (in Japan, Australia, China and Hawaii) and receiver locations together with the areas of the supposed sensitivity for each wave path, are shown.

Two methods have been proposed in order to reveal the amplitude $(A)$ and phase $(P)$ disturbances of VLF signals related to seismic activity. The first of them (chronologically) is based on selecting nighttime bay-like disturbances of $P$ observed over several days before an EQ (Gokhberg et al., 1989; Gufeld et al., 1994; Hayakawa et al., 1994; Gufeld et al., 1992). The second one, the so-called "Terminator Time" (TT) method, makes use of monitoring the location of min- ima of $A$ and $P$ signals during sunrise and sunset (Hayakawa et al., 1996b).

The first step of data processing is the restoration of $P$ continuity, which disturbs itself while crossing the limits $\pm 180^{\circ}$ (Fig. 7). Then, the sliding averaging of $P$ and $A$ records is carried out over $5 \mathrm{~min}$ time intervals. The second step is the calculation of averaged $A$ and $P$ diurnal variations and their dispersions. The third step of data processing is the revealing and if possible the removing of the disturbances with a well-known origin (e.g. X-ray flashes). The last step of data processing is the subtraction of averaged diurnal $A$ and $P$ signals from current signals (Fig. 8).

The disturbances, whose amplitudes exceed $2 \sigma$, can be considered as potential signals of seismogenic origin. An example of such a disturbance is shown in Fig. 8. The beginning of a $P$ disturbance can be seen six days before the Kuril Islands EQ $(M=5.8$, the depth of focus $H=146 \mathrm{~km}$, epicenter distance from wave path $L=3 \mathrm{~km}$ ). The disturbance stopped at about $20 \mathrm{~h}$ before the EQ.

The Terminator method for wave path Japan-Kamchatka was not used for the period of observation.

Observations of $A$ and $P$ values of VLF waves conducted during the time period 1 July 2000 - 30 March 2001 show the following peculiarities:

1. The records of $A$ and $P$ are strongly disturbed both during daytime and nighttime.

2. The correlation of X-rays flashes with $A$ and $P$ disturbances exists during daytime. 


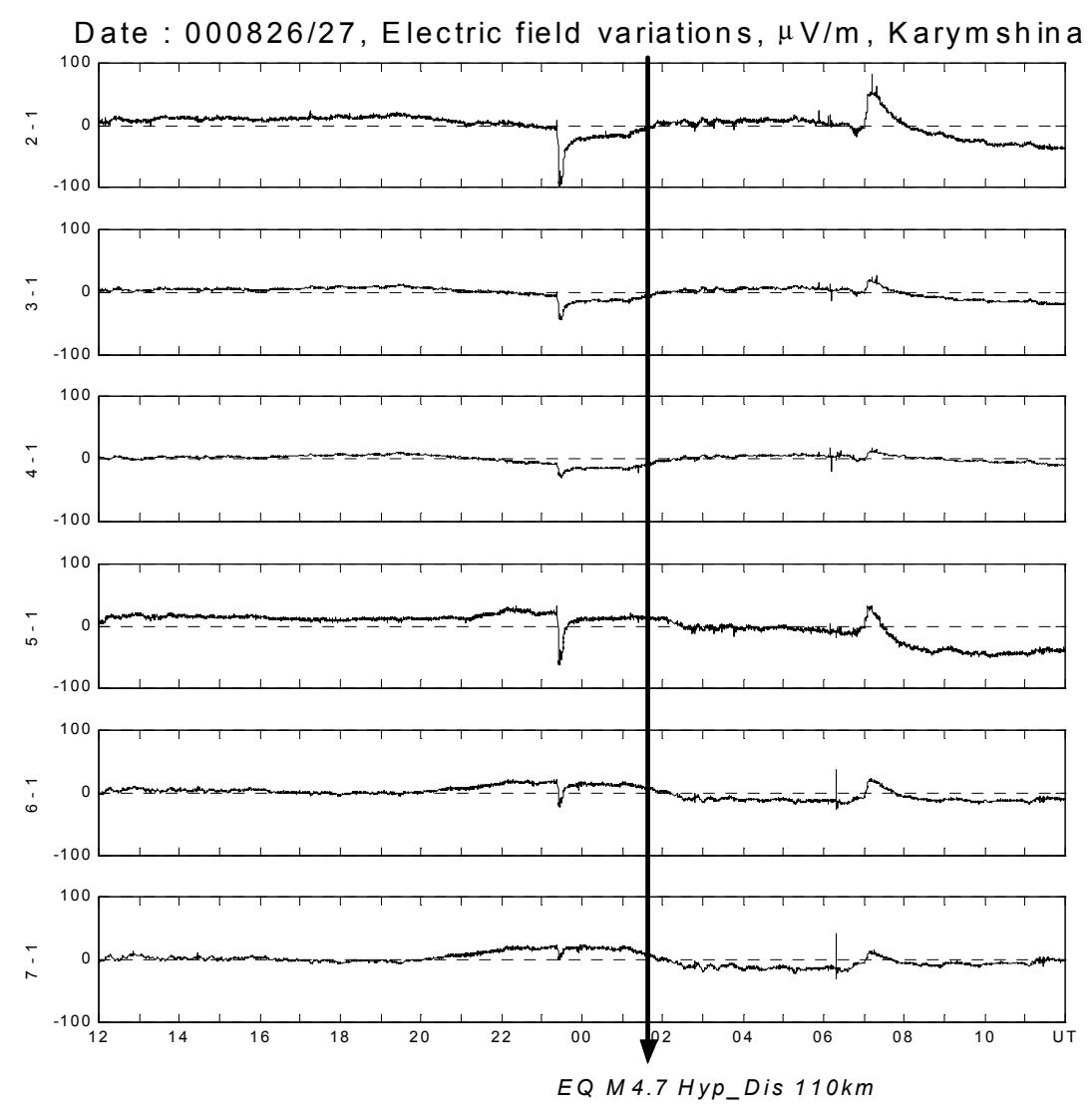

Fig. 9. Geoelectric field variations. The signals possibly resulted from the influence of electrodes or due to the manmade noise.

3. Over all wave paths besides Japan-Kamchatka the trend of the $P$ signal (not always linear) is noticeable. This trend is connected with the low stability of transmitters and complicates the processing of data and their interpretation.

4. Many EQs with $M \geq 5$ are observed along the Japan-Kamchatka wave path, while along the HawaiiKamchatka wave path, the EQs are practically absent. It allows one to compare two records along these paths for better detection of the anomalous behavior of signals along the Japan-Kamchatka path.

5. One can probably interpret the anomalous behaviour of the $P$ record in Fig. 8 as a EQ precursor. The Terminator method cannot be used, since for the time of observations the minimums of the $A$ and $P$ values near the Terminators either have not been found or could be found, but not regularly.

\section{Data analysis of geoelectric potentials (telluric cur- rents)}

The so-called VAN-method of earthquake forecasting, based on the observation of geoelectric potentials (telluric currents) has been put forward by Varotsos and Alexopou- los (1984). According to VAN results, the observed SES (Seismo-Electric Signals) are usually isolated signals, which appear primarily within two to several weeks before the main shock. Typical amplitude of SES for an EQ with $M \sim 5$ achieves $20 \mu \mathrm{V} / \mathrm{m}$ or more at a distance of about $100 \mathrm{~km}$ from the epicenter. The duration of a signal varies from several minutes to several hours. Apparently, the plausibility of this method could be high if the magnitude of the earthquakes is greater than 5.5-5.8 (Mulargia and Gasperini, 1992; Kawase et al., 1993; Hamada, 1993; Uyeda et al., 2000).

According to Uyeda et al. (2001) the equipment is measuring the potential differences $\Delta \mathrm{V}_{2}, \Delta \mathrm{V}_{3}, \Delta \mathrm{V}_{4}$ from NS dipoles of different length $l_{2}=80 \mathrm{~m}, l_{3}=230 \mathrm{~m}, l_{4}=$ $470 \mathrm{~m}$ and potential differences $\Delta \mathrm{V}_{5}, \Delta \mathrm{V}_{6}, \Delta \mathrm{V}_{7}$ from EW dipoles of the same length $l_{5}=80 \mathrm{~m}, l_{6}=230 \mathrm{~m}, l_{7}=$ $470 \mathrm{~m}$. All potential differences $\Delta \mathrm{V}_{i}(i=2,3, \ldots 7)$ were averaged out within $24 \mathrm{~h}$ and then the values $E i=\left(\Delta V_{i}-\right.$ $\left.\left\langle\Delta V_{i}\right\rangle\right) / l_{i}$ were computed for each moment. Here, $\left\langle\Delta V_{i}\right\rangle$ denotes the averaged value of the corresponding potential difference and $E_{i}$ is the estimation of the electric field strength projections on NS or EW directions, respectively.

At the first step of analysis, the signals with amplitude exceeding $30 \mu \mathrm{V} / \mathrm{m}$ and duration over $15 \mathrm{~min}$ were considered as SES. To select signals from different kinds of noise or false SES, we used the original VAN method (Varotsos and 


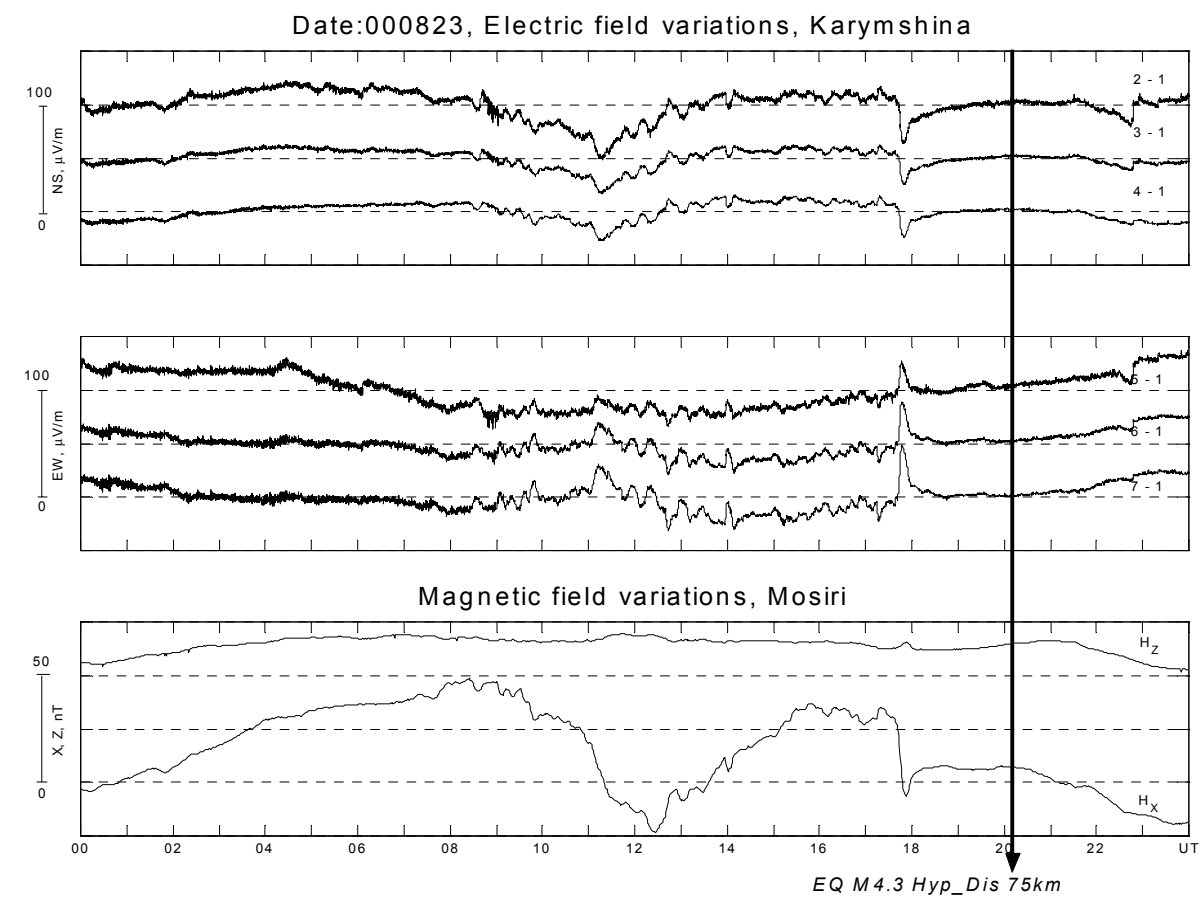

Fig. 10. Geoelectric and magnetic field variations. The signal near $18 \mathrm{~h}$ is possibly of ionospheric or magnetospheric origin.

Lazaridou, 1991).

The registered data involve different kinds of noise or dummy signals that can be conditionally divided into three classes:

1. Industrial or man-made noise,

2. Noise that originates from each electrode,

3. Signals of ionospheric and magnetospheric nature.

If the conductivity of a medium is supposed to be roughly uniform near the measuring system, then the electric field produced by a distant source has to be approximately uniform in the vicinity of a measuring system. The values of $E_{i}$ should be identical for all NS dipoles of this type of field. A similar property has to be valid in the EW direction. The noise from "bad" electrodes and other manmade noise, whose source is near placed, cannot satisfy this condition. To distinguish SES from such noise, a comparison of the values $E_{i}$ was made separately in the NS and EW directions. The example of signals recorded at Karimshino observatory $2 \mathrm{~h}$ before and $5 \mathrm{~h}$ after the earthquake $M=4.7$ on 26-27 August 2000 is shown in Fig. 9. These signals were excluded from SES because of the difference in values of $E_{i}$ recorded by NS dipoles $(i=2-4)$ as well as by EW dipoles $(i=5-7)$.

Some of the signals can be of ionospheric and magnetospheric origin. The low-frequency geomagnetic field variations are global; therefore, these variations can be recorded at many stations simultaneously. Thus, we can identify this effect by analyzing the magnetic data from the neighbouring stations. The example of a signal recorded at Karimshino observatory $2 \mathrm{~h}$ before the earthquake $(M=4.3,23$ August 2000) is shown in Fig. 10. This electric field signal appears simultaneously with the magnetic impulse recorded at the Mosiri (Japan) station and therefore it has possibly a magnetospheric or ionospheric origin.

Thus, during some months since the beginning of the observations, none of the SES-like signals were observed. This fact is not surprising because there were no significant seismic events near the Karimshino observatory within the observation period. At least this fact does not contradict the opinion that SES can be found only for the earthquakes with magnitudes more than 5.5.

\section{References}

Ansari, I. A. and Fraser, B. J.: A multistation study of low latitude Pc3 geomagnetic pulsations, Planet. Space Sci., 34, 519536, 1986.

Bernardi, A., Fraser-Smith, A. C., McGill, P. R., and Villard, Jr., O.G.: ULF magnetic field measurements near the epicenter of the $M_{S}$ 7,1 Loma Prieta earthquake, Phys. Earth Planet. Inter., 68, 1-2, 45-63, 1991.

Dea, J. Y., Hansen, P. M., and Boerner, W. M.: Long-Term ELF Background-Noise Measurements, the Existence of Window Regions, and Applications to Earthquake Precursor Emission Studies, Phys. Earth Planet. Inter., 77, 1-2, 109-125, 1993.

Gokhberg, M. B., Gufeld, I. L., Rozhnoy, A. A., Marenko, V. F., Yampolsky, V. S., and Ponomarev, E. A.: Study of seismic influence on the ionosphere by super long wave probing of the Earth-ionosphere waveguide, Phys. Earth Planet. Inter., 57, 6467, 1989. 
Gorbatikov, A. V., Kodama, T., Molchanov, O. A., and Hayakawa, M.: Long period variations in seismic and electromagnetic measurements, in: Atmospheric and ionospheric electromagnetic phenomena associated with earthquake, Ed. Hayakawa, M., Terra Sci. Pub. Comp., Tokyo, 439-450, 1999.

Gufeld, I. L., Rozhnoy, A. A., Tyumensev, S. N., Sherstuk, S. V., and Ympolsky, V. S.: Radiowave disturbances period to Rudbar and Rachinsk earthquakes, Phys. Earth, 3, 102-106, 1992.

Gufeld, I. L., Gusev, G., and Pokhotelov, O. A.: Is the prediction of earthquake date possible by VLF radio wave monitoring method?, in: Electromagnetic Phenomena Related to Earthquake Prediction, (Eds) Hayakawa, M. and Fujinawa, Y., Terra Sci. Pub. Comp., Tokyo, 381-389, 1994.

Hamada, K.: Statistical evaluation of the SES predictions issued in Greece: Alarm and success rates, Tectonophysics, 224, 203-210, 1993.

Hayakawa, M. and Sato, H.: Ionospheric perturbations associated with earthquakes, as detected by subionospheric VLF propagation, in: Electromagnetic Phenomena Related to Earthquake Prediction, (Eds) Hayakawa, M. and Fujinawa, Y., Terra Sci. Pub. Comp., Tokyo, 391-397, 1994.

Hayakawa, M., Kawate, R., Molchanov, O. A., and Yumoto, K.: Results of ultra-low-frequency magnetic field measurements during the Guam earthquake of 8 August 1993, Geophys. Res. Lett., 23, 241-244, 1996a.

Hayakawa, M., Molchanov, O. A., Ondoh, T., and Kawai, E.: Precursory Signature of the Kobe Earthquake on VLF Subionospheric Signal, J. Atmos. Electr., 16, 3, 247-257, 1996b.

Kawase, T., Uyeda, S., Uyeshima, M., and Kinoshita, M.: Possible correlation between geoelectric potential change in IzuOshima island and the earthquake swarm off the East Izu Peninsula, Japan, Tectonophysics, 224, 1/3, 83-93, 1993.

Kopytenko, Yu. A., Matiashvily, T. G., Voronov, P. M., Kopytenko, E. A., and Molchanov, O. A.: Detection of ULF emission con- nected with the Spitak earthquake and its aftershock activity based on geomagnetic pulsations data at Dusheti and Vardziya observatories, Phys. Earth and Plan. Int., 77, 85-95, 1993.

Molchanov, O. A., Kulchitsky, A., and Hayakawa, M.: ULF emission due to inductive seismo-electromagnetic effect, in: Electromagnetic Phenomena Related to Earthquake Prediction, (Eds) Hayakawa, M. and Molchanov, O., Terra Sci. Pub. Comp., Tokyo, 2001.

Mulargia, F. and Gasperini, P.: Evaluating the statistical validity beyond chance of VAN earthquake precursors, Geophys. J. Int., 111, 1, 32-44, 1992.

Pudovkin, M., Raspopov, O., and Kleimenova, N.: Disturbances of electromagnetic Earth's Field. Part II: Short-period variations of geomagnetic field, Leningrad University, Leningrad, 1976.

Samson, J. C., Jacob, J. A., and Rostoker, G.: Latitude dependent characteristics of long period geomagnetic pulsation, J. Geophys. Res., 76, 3675-3683, 1971.

Uyeda, S., Nagao, T., Orihara, Y., Yamaguchi, T., and Takahashi, I.: Geoelectric potential changes: Possible precursors to earthquakes in Japan, Proc. Nat. Acad. Sci., 97, 4561-4566, 2000.

Uyeda, S., Nagao, T., Hattori, K., Noda, Y., Hayakawa, M., Miyaki, K., Molchanov, O., Gladychev, V., Baransky, L., Schekotov, A., Belyaev, G., Fedorov, E., Pokhotelov, O., Andreevsky, S., Rozhnoi, A., Khabazin, Y., Gorbatikov, A., Gordeev, E., Chebrov, V., Lutikov, A., Yunga, S., Kosarev, G., and Surkov, V.: Geophysical Observatory in Kamchatka region for monitoring of phenomena connected with seismic activity, Nat. Hazards and Earth Sc. Sciences, 1, 1/2, 3-7, 2001.

Varotsos, P. and Alexopoulos, K.: Physical properties of the variations of the electric field of the earth preceding earthquake II, Tectonophysics, 110, 1/3, 99-125, 1984.

Varotsos, P. and Lazaridou, M.: Latest aspects of earthquake prediction and electric signals, Tectonophysics, 188, 321-347, 1991. 